Appl. Set-Valued Anal. Optim. 1 (2019), No. 3, pp. 205-219

Available online at http://asvao.biemdas.com

https://doi.org/10.23952/asvao.1.2019.3.02

\title{
ON STRICTLY MINIMAL ELEMENTS W.R.T. PREORDER RELATIONS IN SET-VALUED OPTIMIZATION
}

\author{
CHRISTIAN GÜNTHER ${ }^{1,2, *}$, ELISABETH KÖBIS ${ }^{2}$, NICOLAE POPOVICI $^{3}$ \\ ${ }^{1}$ Faculty of Natural Sciences III, Institute of Informatics, \\ Martin Luther University Halle-Wittenberg, Halle, Germany \\ ${ }^{2}$ Faculty of Natural Sciences II, Institute of Mathematics, \\ Martin Luther University Halle-Wittenberg, Halle, Germany \\ ${ }^{3}$ Faculty of Mathematics and Computer Science, Babeş-Bolyai University, 400084 Cluj-Napoca, Romania
}

\begin{abstract}
The principal aim of this paper is to develop two algorithms for computing all strictly minimal elements of a nonempty finite family of sets in a real linear space, with respect to a preorder relation defined on the power set of that space. By implementing these algorithms in MATLAB we compute all strictly minimal elements of some test families of rectangles, with respect to $\ell$-type and $u$-type preorder relations induced by the standard ordering cone in the Euclidean plane.
\end{abstract}

Keywords. Preorder relation; strictly minimal element; external stability; Graef-Younes reduction method; sorting scalar function; vector optimization; set optimization.

2010 Mathematics Subject Classification. 49J53, 46A40, 90C29.

\section{INTRODUCTION}

Different concepts of optimality are currently known in set optimization and various types of binary relations can be used in order to define them (see, e.g., Eichfelder and Pilecka [5, 6], Jahn and Ha [13], Khan, Tammer and Zălinescu [15], Köbis and Le [17], Kuroiwa [18]). In particular, by considering a real linear space $E$, a nonempty family of sets $\mathscr{A} \subseteq \mathscr{P}(E)$, and a preorder (i.e., reflexive and transitive) relation $\preccurlyeq$ on $\mathscr{A}$, an element $A \in \mathscr{A}$ is said to be a:

- minimal element of $\mathscr{A}$ w.r.t. $\preccurlyeq$, if for any $A^{\prime} \in \mathscr{A}$ such that $A^{\prime} \preccurlyeq A$ we have $A \preccurlyeq A^{\prime}$;

- strictly minimal element of $\mathscr{A}$ w.r.t. $\preccurlyeq$, if for any $A^{\prime} \in \mathscr{A}$ such that $A^{\prime} \preccurlyeq A$ we have $A=A^{\prime}$. Of course, these two concepts coincide when $\preccurlyeq$ is antisymmetric, but this is not always the case.

An interesting and challenging topic in set optimization is to develop effective methods to compute all (strictly) minimal elements of $\mathscr{A}$ w.r.t. $\preccurlyeq$. Recently, Köbis, Kuroiwa and Tammer [16] have proposed an algorithm for computing all minimal elements of a finite family of sets $\mathscr{A}$ w.r.t. a preorder relation $\preccurlyeq$. A refined variant of this algorithm has been presented by us in [8]. Köbis and Le [17] have proposed algorithms for computing the so-called "strong minimal solutions" of an optimization problem involving a set-valued objective function $F$ and a finite

\footnotetext{
${ }^{*}$ Corresponding author.

E-mail addresses: christian.guenther@mathematik.uni-halle.de (C. Günther), elisabeth.koebis@mathematik. uni-halle.de (E. Köbis), popovici@math.ubbcluj.ro (N. Popovici).

Received October 24, 2019; Accepted November 27, 2019.
} 
feasible set $S$, which in terms of outcomes reduces to compute all strictly minimal elements of the finite family of sets $\mathscr{A}:=\{F(x) \mid x \in S\}$. All these algorithms are mainly based on certain set-valued counterparts of some methods originally conceived for vector optimization problems, such as the well-known Graef-Younes reduction procedure and Jahn-Graef-Younes method (see, e.g., Eichfelder [1, 2, 3], Günther and Popovici [9], Jahn [10, 11], Jahn and Rathje [14], Younes [22], and the references therein).

Our principal aim is to develop new algorithms for computing all strictly minimal elements of a finite family of sets $\mathscr{A}$ w.r.t. a preorder relation $\preccurlyeq$, that are shown to be implementable for certain families of rectangles in the Euclidean plane while using the $\ell$-type and $u$-type preorder relations (in the sense of Kuroiwa [18]) induced by the standard ordering cone.

The paper is organized as follows. In Section 2 we present some preliminary results concerning minimal and strictly minimal elements of a family $\mathscr{A}$ of sets in a real linear space $E$, with respect to a preorder relation $\preccurlyeq$. In particular, we study the external stability of the sets $\mathrm{MIN}_{\preccurlyeq}(\mathscr{A})$ and $\mathrm{SMIN}_{\preccurlyeq}(\mathscr{A})$ of all minimal and strictly minimal elements of $\mathscr{A}$ w.r.t. $\preccurlyeq$, as well as the role of (strongly) $\preccurlyeq$-increasing real-valued functions in generating some minimal and strictly minimal elements of $\mathscr{A}$.

In Section 3, we investigate the minimal elements as well as the strictly minimal elements of a family of sets $\mathscr{A}$ with respect to the $\ell$-type and $u$-type preorder relations, $\preccurlyeq_{C}^{\ell}$ and $\preccurlyeq_{C}^{u}$, induced by a convex cone $C \subseteq E$. In particular, we emphasize the connection with optimality concepts in vector optimization.

Section 4 represents the main part of the paper, being devoted to the computation of the set $\mathrm{SMIN}_{\preccurlyeq}(\mathscr{A})$, where $\mathscr{A}$ is assumed to be finite. First we recall a method for computing the set $\mathrm{MIN}_{\preccurlyeq}(\mathscr{A})$, namely Algorithm 1, which was developed by us in [8]. Then, we present two methods for computing the set $\mathrm{SMIN}_{\preccurlyeq}(\mathscr{A})$ : Algorithm 2, which is similar to some methods proposed by Eichfelder [2, 3], Köbis and Le [17], and Algorithm 3, which consists in computing $\mathrm{MIN}_{\preccurlyeq}(\mathscr{A})$ by Algorithm 1 and thereafter $\operatorname{SMIN}_{\preccurlyeq}\left(\mathrm{MIN}_{\preccurlyeq}(\mathscr{A})\right)$ by Algorithm 2 .

Numerical experiments in MATLAB are provided in Section 5 along with a comparative analysis of Algorithms 1, 2 and 3.

Section 6 contains some concluding remarks and further research directions.

\section{Minimality AND STRICT Minimality W.R.T. GENERAL PREORDER RELATIONS}

Throughout this paper $E$ denotes a real linear space and $\mathscr{A} \subseteq \mathscr{P}(E)$ is a nonempty family of subsets of $E$. We assume that $\mathscr{A}$ is endowed with a preorder relation $\preccurlyeq$, which means that

- $\preccurlyeq$ is reflexive (i.e., $\forall A \in \mathscr{A}: A \preccurlyeq A$ ) and

- $\preccurlyeq$ is transitive (i.e., $\forall A, A^{\prime}, A^{\prime \prime} \in \mathscr{A}: A^{\prime} \preccurlyeq A$ and $A^{\prime \prime} \preccurlyeq A^{\prime} \Longrightarrow A^{\prime \prime} \preccurlyeq A$ ).

Definition 2.1. We say that $A \in \mathscr{A}$ is a minimal element of $\mathscr{A}$ (w.r.t. $\preccurlyeq$ ) if

$$
\forall A^{\prime} \in \mathscr{A}: A^{\prime} \preccurlyeq A \Longrightarrow A \preccurlyeq A^{\prime}
$$

We denote by $\operatorname{MIN}_{\preccurlyeq}(\mathscr{A})$ the set of all minimal elements of $\mathscr{A}$, i.e.,

$$
\begin{aligned}
\operatorname{MIN}_{\preccurlyeq}(\mathscr{A}) & =\left\{A \in \mathscr{A} \mid \forall A^{\prime} \in \mathscr{A}: A^{\prime} \preccurlyeq A \Longrightarrow A \preccurlyeq A^{\prime}\right\} \\
& =\left\{A \in \mathscr{A} \mid \nexists A^{\prime} \in \mathscr{A} \backslash\{A\}: A^{\prime} \preccurlyeq A \text { and } A \npreceq A^{\prime}\right\} .
\end{aligned}
$$


Definition 2.2. We say that $A \in \mathscr{A}$ is a strictly minimal element of $\mathscr{A}$ (w.r.t. $\preccurlyeq$ ) if

$$
\forall A^{\prime} \in \mathscr{A}: A^{\prime} \preccurlyeq A \Longrightarrow A=A^{\prime} .
$$

We denote by $\operatorname{SMIN}_{\preccurlyeq}(\mathscr{A})$ the set of all strictly minimal elements of $\mathscr{A}$, i.e.,

$$
\begin{aligned}
\operatorname{SMIN}_{\preccurlyeq}(\mathscr{A}) & =\left\{A \in \mathscr{A} \mid \forall A^{\prime} \in \mathscr{A}: A^{\prime} \preccurlyeq A \Longrightarrow A=A^{\prime}\right\} \\
& =\left\{A \in \mathscr{A} \mid \nexists A^{\prime} \in \mathscr{A} \backslash\{A\}: A^{\prime} \preccurlyeq A\right\} .
\end{aligned}
$$

Similarly, for any nonempty family $\mathscr{B} \subseteq \mathscr{A}$ we denote by

$$
\operatorname{MIN}_{\preccurlyeq}(\mathscr{B}) \text { and } \operatorname{SMIN}_{\preccurlyeq}(\mathscr{B})
$$

the sets of all minimal and strictly minimal elements of $\mathscr{B}$ (w.r.t. $\preccurlyeq$ restricted to $\mathscr{B}$ ).

Remark 2.1. a) By reflexivity of $\preccurlyeq$ it is easily seen that

$$
\operatorname{SMIN}_{\preccurlyeq}(\mathscr{A}) \subseteq \operatorname{MIN}_{\preccurlyeq}(\mathscr{A}) \text {. }
$$

b) If $\preccurlyeq$ is antisymmetric (hence is an order relation), i.e.,

$$
\forall A, A^{\prime} \in \mathscr{A}: A^{\prime} \preccurlyeq A \text { and } A \preccurlyeq A^{\prime} \Longrightarrow A=A^{\prime},
$$

then every minimal element of $\mathscr{A}$ is actually strictly minimal, i.e.,

$$
\mathrm{MIN}_{\preccurlyeq}(\mathscr{A})=\operatorname{SMIN}_{\preccurlyeq}(\mathscr{A}) \text {. }
$$

c) In general, the equality $\operatorname{MIN}_{\preccurlyeq}(\mathscr{A})=\operatorname{SMIN}_{\preccurlyeq}(\mathscr{A})$ does not imply the antisymmetry of $\preccurlyeq$, as we will see in Example 3.1.

Lemma 2.1. It is easy to see that for any nonempty family $\mathscr{B} \subseteq \mathscr{A}$ we have

$$
\mathscr{B} \cap \operatorname{SMIN}_{\preccurlyeq}(\mathscr{A}) \subseteq \operatorname{SMIN}_{\preccurlyeq}(\mathscr{B}),
$$

by definition of strictly minimal elements of $\mathscr{A}$ and $\mathscr{B}$.

Theorem 2.1. If $\mathrm{MIN}_{\preccurlyeq}(\mathscr{A})$ is nonempty, then

$$
\operatorname{SMIN}_{\preccurlyeq}(\mathscr{A})=\operatorname{SMIN}_{\preccurlyeq}\left(\operatorname{MIN}_{\preccurlyeq}(\mathscr{A})\right) .
$$

Proof. The inclusion " $\subseteq$ " in (2.1) holds by Lemma 2.1 applied to $\mathscr{B}=\operatorname{MIN}_{\preccurlyeq}(\mathscr{A})$. In what concerns the converse inclusion " $\supseteq$ ", let any $A \in \mathrm{SMIN}_{\preccurlyeq}\left(\mathrm{MIN}_{\preccurlyeq}(\mathscr{A})\right)$. In order to show that $A \in \mathrm{SMIN}_{\preccurlyeq}(\mathscr{A})$, consider any $A^{\prime} \in \mathscr{A}$ such that

$$
A^{\prime} \preccurlyeq A \text {. }
$$

We just have to prove that

$$
A^{\prime}=A
$$

To this aim, we first show that

$$
A^{\prime} \in \operatorname{MIN}_{\preccurlyeq}(\mathscr{A}) .
$$

Indeed, let $A^{\prime \prime} \in \mathscr{A}$ be such that $A^{\prime \prime} \preccurlyeq A^{\prime}$. Recalling that $A^{\prime} \preccurlyeq A$, we infer by transitivity of $\preccurlyeq$ that $A^{\prime \prime} \preccurlyeq A$. Therefore, taking into account that $A \in \mathrm{MIN}_{\preccurlyeq}(\mathscr{A})$ (since $A \in \mathrm{SMIN}_{\preccurlyeq}\left(\mathrm{MIN}_{\preccurlyeq}(\mathscr{A})\right)$ ), we can deduce that $A \preccurlyeq A^{\prime \prime}$. Recalling (2.2), we infer by transitivity of $\preccurlyeq$ that $A^{\prime} \preccurlyeq A^{\prime \prime}$. Thus (2.4) holds true. Finally, by (2.2), (2.4) and the fact that $A \in \operatorname{SMIN}_{\preccurlyeq}\left(\operatorname{MIN}_{\preccurlyeq}(\mathscr{A})\right)$, we get the desired equality (2.3). 
Definition 2.3. We say that a family $\mathscr{B} \subseteq \mathscr{A}$ is externally stable (in $\mathscr{A}$ w.r.t. $\preccurlyeq$ ) if

$$
\forall A \in \mathscr{A} \backslash \mathscr{B}, \exists B \in \mathscr{B}: B \preccurlyeq A .
$$

Remark 2.2. If a family $\mathscr{B} \subseteq \mathscr{A}$ is externally stable, then $\mathscr{B}$ is nonempty.

Theorem 2.2. If $\mathrm{SMIN}_{\preccurlyeq}(\mathscr{A})$ is externally stable, then

$$
\operatorname{SMIN}_{\preccurlyeq}(\mathscr{A})=\operatorname{MIN}_{\preccurlyeq}(\mathscr{A}) .
$$

Proof. In view of Remark 2.1 (a), we just have to prove that

$$
\operatorname{MIN}_{\preccurlyeq}(\mathscr{A}) \subseteq \operatorname{SMIN}_{\preccurlyeq}(\mathscr{A}) .
$$

Suppose by the contrary that there exists some $A \in \operatorname{MIN}_{\preccurlyeq}(\mathscr{A}) \backslash \operatorname{SMIN}_{\preccurlyeq}(\mathscr{A})$. Then, by external stability of $\operatorname{SMIN}_{\preccurlyeq}(\mathscr{A})$, we find $A^{\prime} \in \operatorname{SMIN}_{\preccurlyeq}(\mathscr{A})$ such that $A^{\prime} \preccurlyeq A$. Since $A \in \mathrm{MIN}_{\preccurlyeq}(\mathscr{A})$, we infer that $A \preccurlyeq A^{\prime}$. Now, taking into account that $A^{\prime} \in \operatorname{SMIN}_{\preccurlyeq}(\mathscr{A})$, it follows that $A^{\prime}=A$, hence $A \in \mathrm{SMIN}_{\preccurlyeq}(\mathscr{A})$, a contradiction.

Remark 2.3. It is well-known that if $\mathscr{A}$ is finite then the $\mathrm{MIN}_{\preccurlyeq}(\mathscr{A})$ is externally stable (see, e.g., Podinovskiı and Nogin [20, p. 21]).

In view of Remarks 2.1 (b) and 2.3, we obtain the following result.

Proposition 2.1. Assume that $\mathscr{A}$ is finite. If $\operatorname{SMIN}_{\preccurlyeq}(\mathscr{A})=\mathrm{MIN}_{\preccurlyeq}(\mathscr{A})$ (in particular, if $\preccurlyeq$ is antisymmetric), then $\mathrm{SMIN}_{\preccurlyeq}(\mathscr{A})$ is externally stable.

Definition 2.4. A scalar function $\varphi: \mathscr{A} \rightarrow \mathbb{R}$ is said to be:

- $\preccurlyeq$-increasing, if for any $A, A^{\prime} \in \mathscr{A}$,

$$
A^{\prime} \preccurlyeq A \Longrightarrow \varphi\left(A^{\prime}\right) \leq \varphi(A) ;
$$

- strongly $\preccurlyeq$-increasing, if for any $A, A^{\prime} \in \mathscr{A}$,

$$
A^{\prime} \preccurlyeq A \text { and } A \npreceq A^{\prime} \Longrightarrow \varphi\left(A^{\prime}\right)<\varphi(A) ;
$$

Lemma 2.2. Let $\varphi: \mathscr{A} \rightarrow \mathbb{R}$ be a function and let

$$
\operatorname{argmin}_{A \in \mathscr{A}} \varphi(A):=\left\{A^{\prime} \in \mathscr{A} \mid \forall A \in \mathscr{A}: \varphi\left(A^{\prime}\right) \leq \varphi(A)\right\}
$$

be the set of its minimizers. The following assertions hold:

$1^{\circ}$ If $\varphi$ is strongly $\preccurlyeq$-increasing, then

$$
\operatorname{argmin}_{A \in \mathscr{A}} \varphi(A) \subseteq \operatorname{MIN}_{\preccurlyeq}(\mathscr{A}) .
$$

$2^{\circ}$ If either

(i) $\varphi$ is strongly $\preccurlyeq$-increasing and $\preccurlyeq$ is antisymmetric or

(ii) $\varphi$ is $\preccurlyeq$-increasing and $\operatorname{argmin}_{A \in \mathscr{A}} \varphi(A)$ is a singleton, then we have

$$
\operatorname{argmin}_{A \in \mathscr{A}} \varphi(A) \subseteq \mathrm{SMIN}_{\preccurlyeq}(\mathscr{A}) .
$$

Proof. Assertion $1^{\circ}$ is well-known (see, e.g., Günther, Köbis and Popovici [8], or Podinovskiŭ and Nogin [20] for a more general framework).

In what concerns assertion $2^{\circ}$, consider any $A^{\prime} \in \operatorname{argmin}_{A \in \mathscr{A}} \varphi(A)$. Assume by the contrary that $A^{\prime} \notin \operatorname{SMIN}_{\preccurlyeq}(\mathscr{A})$. Then, there exists $A^{\prime \prime} \in \mathscr{A}$ with $A^{\prime \prime} \preccurlyeq A^{\prime}$ and $A^{\prime} \neq A^{\prime \prime}$. 
(i) Assume that $\varphi$ is strongly $\preccurlyeq$-increasing while $\preccurlyeq$ is antisymmetric. In this case, by $1^{\circ}$ we have $A^{\prime} \in \mathrm{MIN}_{\preccurlyeq}(\mathscr{A})$. Therefore, since $A^{\prime \prime} \preccurlyeq A^{\prime}$, it follows that $A^{\prime} \preccurlyeq A^{\prime \prime}$. The antisymmetry of $\preccurlyeq$ entails $A^{\prime}=A^{\prime \prime}$, a contradiction.

(ii) Assume that $\varphi$ is $\preccurlyeq$-increasing and $\operatorname{argmin}_{A \in \mathscr{A}} \varphi(A)$ is a singleton. Since $A^{\prime \prime} \preccurlyeq A^{\prime}$, we have $\varphi\left(A^{\prime \prime}\right) \leq \varphi\left(A^{\prime}\right)$, hence $A^{\prime \prime}$ is a minimizer of $\varphi$. Recalling that $\operatorname{argmin}_{A \in \mathscr{A}} \varphi(A)$ is a singleton, we deduce that $A^{\prime}=A^{\prime \prime}$, a contradiction.

\section{Minimality AND STRICT MINIMALITY W.R.T. $\ell$-TYPE AND $u$-TYPE PREORDER RELATIONS}

Let $C \subseteq E$ be a convex cone, i.e., $0 \in C=\mathbb{R}_{+} \cdot C=C+C$, where 0 stands for the origin of $E$. As usual in vector optimization (see, e.g., Göpfert et al. [7], Jahn [11] and Luc [19]), for any nonempty set $A \in \mathscr{P}(E)$, we define the sets of minimal and maximal elements of $A$ w.r.t. $C$ by

$$
\begin{aligned}
& \min _{C}(A)=\{x \in A \mid(x-C) \cap A \subseteq x+C\} ; \\
& \max _{C}(A)=\{x \in A \mid(x+C) \cap A \subseteq x-C\} .
\end{aligned}
$$

Within set optimization one can define different types of binary relations on $\mathscr{P}(E)$ by means of $C$ (see, e.g., Jahn and Ha [13], Khan, Tammer, Zălinescu [15], Kuroiwa [18] and Seto, Kuroiwa, Popovici [21]). In what follows we recall two of them, namely the preorder relations $\preccurlyeq_{C}^{\ell}$ and $\preccurlyeq_{C}^{u}$, defined for any $A, A^{\prime} \in \mathscr{P}(E)$ by

$$
\begin{aligned}
& A^{\prime} \preccurlyeq_{C}^{\ell} A: \Longleftrightarrow A \subseteq A^{\prime}+C \Longleftrightarrow A+C \subseteq A^{\prime}+C ; \\
& A^{\prime} \preccurlyeq_{C}^{u} A: \Longleftrightarrow A^{\prime} \subseteq A-C \Longleftrightarrow A^{\prime}-C \subseteq A-C .
\end{aligned}
$$

By considering $\preccurlyeq_{C}^{\ell}$ and $\preccurlyeq_{C}^{u}$ in the role of $\preccurlyeq$ in Definitions 2.1 and 2.2 we obtain

$$
\begin{aligned}
\operatorname{MIN}_{\preccurlyeq_{C}^{\ell}}(\mathscr{A}) & =\left\{A \in \mathscr{A} \mid \forall A^{\prime} \in \mathscr{A}: A \subseteq A^{\prime}+C \Longrightarrow A^{\prime} \subseteq A+C\right\} \\
& =\left\{A \in \mathscr{A} \mid \nexists A^{\prime} \in \mathscr{A} \backslash\{A\}: A+C \subseteq A^{\prime}+C \text { and } A+C \neq A^{\prime}+C\right\} ; \\
\operatorname{MIN}_{\preccurlyeq_{C}^{u}}(\mathscr{A}) & =\left\{A \in \mathscr{A} \mid \forall A^{\prime} \in \mathscr{A}: A^{\prime} \subseteq A-C \Longrightarrow A \subseteq A^{\prime}-C\right\} \\
& =\left\{A \in \mathscr{A} \mid \nexists A^{\prime} \in \mathscr{A} \backslash\{A\}: A^{\prime}-C \subseteq A-C \text { and } A-C \neq A^{\prime}-C\right\} ; \\
\operatorname{SMIN}_{\preccurlyeq_{C}^{\ell}}(\mathscr{A}) & =\left\{A \in \mathscr{A} \mid \forall A^{\prime} \in \mathscr{A}: A \subseteq A^{\prime}+C \Longrightarrow A=A^{\prime}\right\} \\
& =\left\{A \in \mathscr{A} \mid \nexists A^{\prime} \in \mathscr{A} \backslash\{A\}: A+C \subseteq A^{\prime}+C\right\} ; \\
\operatorname{SMIN}_{\preccurlyeq_{C}^{u}}(\mathscr{A}) & =\left\{A \in \mathscr{A} \mid \forall A^{\prime} \in \mathscr{A}: A^{\prime} \subseteq A-C \Longrightarrow A=A^{\prime}\right\} \\
& =\left\{A \in \mathscr{A} \mid \nexists A^{\prime} \in \mathscr{A} \backslash\{A\}: A^{\prime}-C \subseteq A-C\right\} .
\end{aligned}
$$

Remark 3.1. If the sets $A, A^{\prime} \in \mathscr{P}(E)$ satisfy the following conditions

$$
\begin{aligned}
A \subseteq \min _{C}(A)+C, \text { i.e., } A+C=\min _{C}(A)+C, \\
A^{\prime} \subseteq \min _{C}\left(A^{\prime}\right)+C, \text { i.e., } A^{\prime}+C=\min _{C}\left(A^{\prime}\right)+C, \\
A \subseteq \max _{C}(A)-C, \text { i.e., } A-C=\max _{C}(A)-C, \\
A^{\prime} \subseteq \max _{C}\left(A^{\prime}\right)-C, \text { i.e., } A^{\prime}-C=\max _{C}\left(A^{\prime}\right)-C,
\end{aligned}
$$


(in other words, if the domination property holds, cf. Luc [19]), then it is easily seen that

$$
\begin{aligned}
& A^{\prime} \preccurlyeq_{C}^{\ell} A \Longleftrightarrow \min _{C}\left(A^{\prime}\right) \preccurlyeq_{C}^{\ell} \min _{C}(A) ; \\
& A^{\prime} \preccurlyeq_{C}^{u} A \Longleftrightarrow \max _{C}\left(A^{\prime}\right) \preccurlyeq_{C}^{u} \max _{C}(A) .
\end{aligned}
$$

Example 3.1. Let us consider the particular framework where $E=\mathbb{R}^{2}$ is endowed with the standard ordering cone $C=\mathbb{R}_{+}^{2}$ and let $\mathscr{A}=\left\{A^{1}, \ldots, A^{7}\right\}$ be the family of sets represented in Figure 1, namely the rectangles/squares $A^{1}, A^{2}, A^{3}, A^{4}$ and the line segments $A^{5}, A^{6}, A^{7}$.

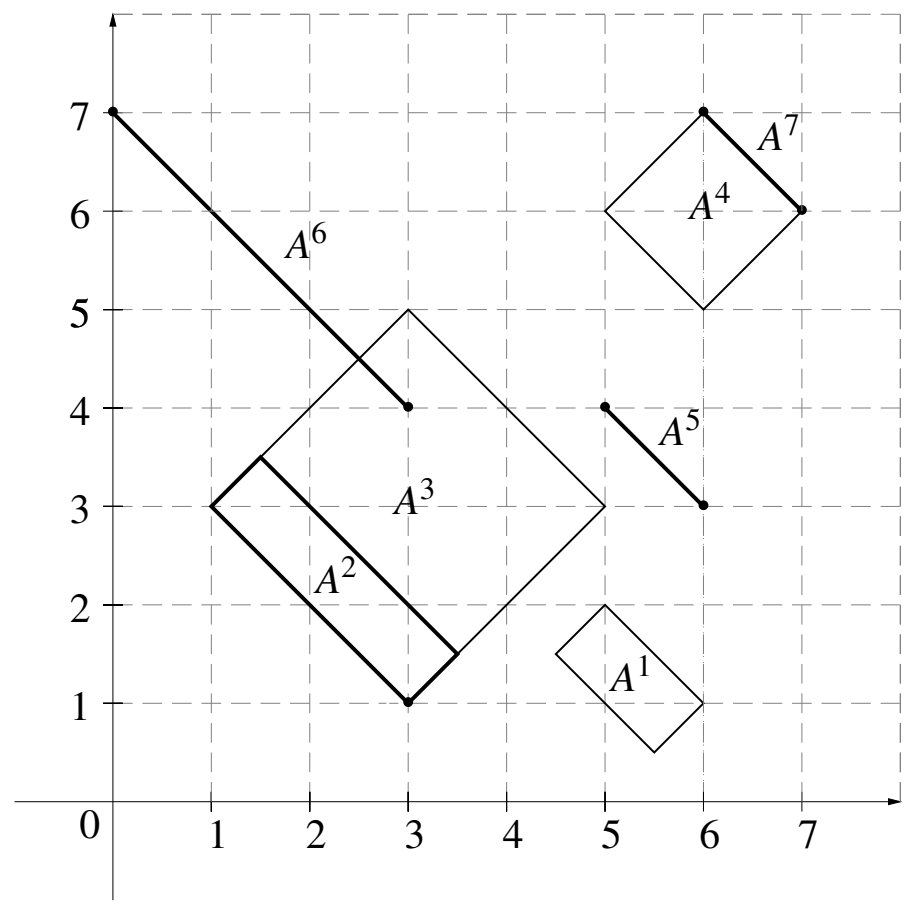

Figure 1. The family $\mathscr{A}$ in Example 3.1.

It is a simple exercise to check that

$$
\begin{aligned}
\operatorname{MIN}_{\preccurlyeq_{C}^{\ell}}(\mathscr{A}) & =\left\{A^{1}, A^{2}, A^{3}, A^{6}\right\}, & \operatorname{MiN}_{\preccurlyeq}{ }_{C}^{u}(\mathscr{A}) & =\left\{A^{1}, A^{2}, A^{6}\right\}, \\
\operatorname{SMIN}_{\preccurlyeq_{C}^{\ell}}(\mathscr{A}) & =\left\{A^{1}, A^{6}\right\}, & \operatorname{SMIN}_{u}(\mathscr{A}) & =\left\{A^{1}, A^{2}, A^{6}\right\} .
\end{aligned}
$$

Since $\operatorname{MIN}_{\preccurlyeq l}(\mathscr{A}) \neq \operatorname{SMIN}_{\preccurlyeq l}(\mathscr{A})$, the relation $\preccurlyeq_{C}^{\ell}$ is not antisymmetric, in view of Remark 2.1 (b). Also, although $\operatorname{MIN}_{\preccurlyeq C}(\mathscr{A})=\operatorname{SMIN}_{\preccurlyeq}{ }_{C}^{u}(\mathscr{A})$, the relation $\preccurlyeq_{C}^{u}$ is not antisymmetric, because we have $A^{4} \preccurlyeq_{C}^{u} A^{7}$ and $A^{7} \preccurlyeq_{C}^{u} A^{4}$, while $A^{4} \neq A^{7}$. Notice that the sets $\operatorname{MIN}_{\preccurlyeq}(\mathscr{A})$ and $\operatorname{MIN}_{\preccurlyeq} \preccurlyeq_{C}^{u}(\mathscr{A})=\operatorname{SMIN}_{\preccurlyeq}{ }_{C}(\mathscr{A})$ are externally stable in view of Remark 2.3, but the set $\mathrm{SMIN}_{\preccurlyeq}(\mathscr{A})$ is not externally stable, since for $A:=A^{2} \in \mathscr{A} \backslash \operatorname{SMIN}_{\preccurlyeq_{C}^{\ell}}(\mathscr{A})$ there is no $B \in \operatorname{SMIN}_{\preccurlyeq}(\mathscr{A})$ such that $B \preccurlyeq_{C}^{\ell} A$.

\section{Algorithms for COMputing the Set $\operatorname{SMIN}_{\preccurlyeq}(\mathscr{A})$ WHEN $\mathscr{A}$ IS Finite}

Throughout this section, we assume that

$$
\mathscr{A}=\left\{A^{1}, \ldots, A^{p}\right\} \subseteq \mathscr{P}(E)
$$


is a finite family of sets, where $p \in \mathbb{N}, p \geq 2$.

The following algorithm, proposed by us in [8], is a reduction procedure that eliminates some of the non-strictly minimal elements of $\mathscr{A}$ w.r.t. $\preccurlyeq$, and therefore it provides a family

$$
\mathscr{B}=\operatorname{MIN}_{\preccurlyeq}(\mathscr{A}) \subseteq \mathscr{A},
$$

which still contains all strictly minimal elements of $\mathscr{A}$ in view of Remark 2.1 (a).

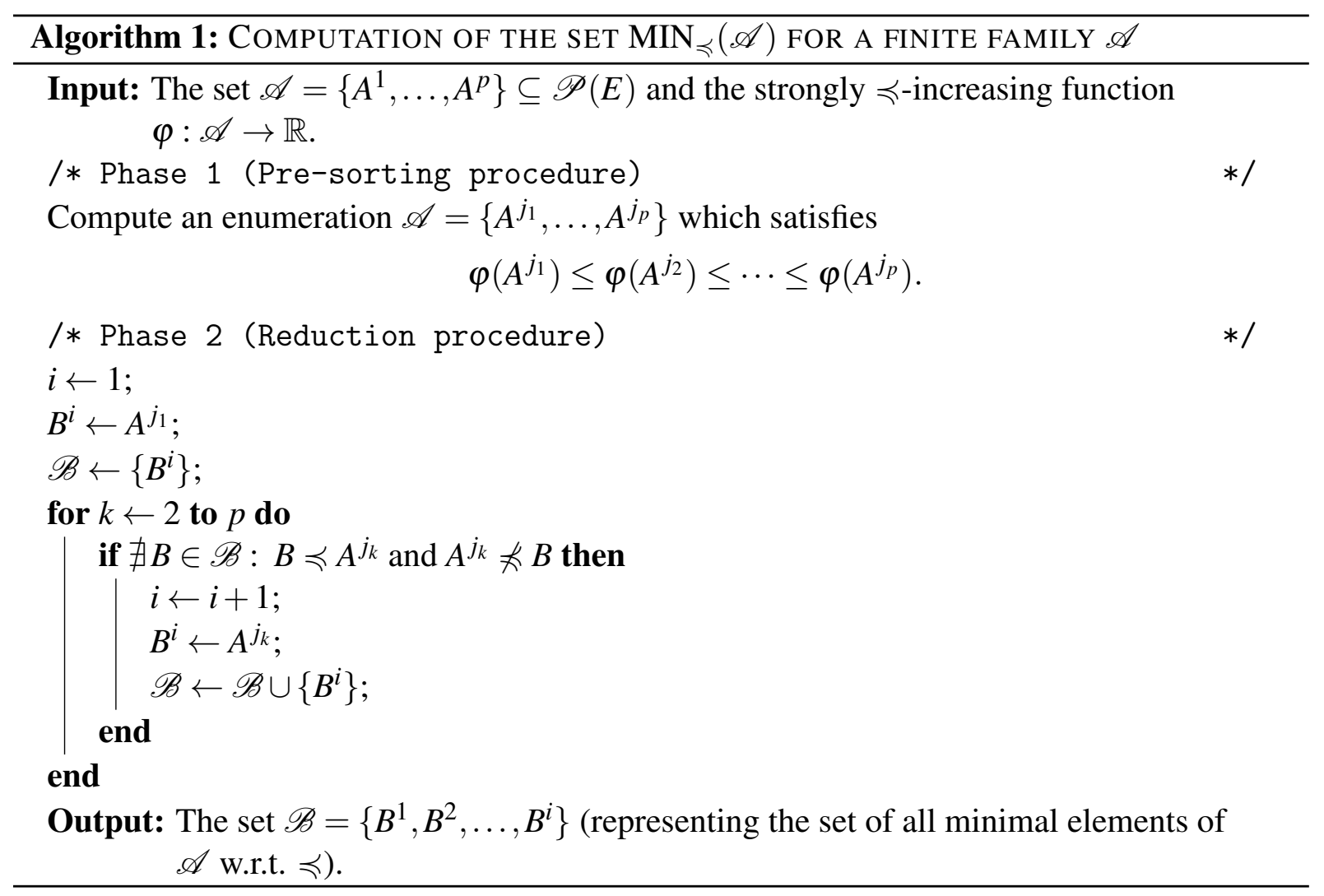

Remark 4.1. As shown by us in [8], in the worst-case scenario, Algorithm 1 needs to perform

$$
|\mathscr{B}| \cdot(2|\mathscr{A}|-|\mathscr{B}|-1) / 2
$$

pairwise comparisons of elements w.r.t. $\preccurlyeq$ (where, a comparison of two sets $A, A^{\prime} \in \mathscr{A}$ consists in checking both conditions $A^{\prime} \preccurlyeq A$ and $A \npreceq A^{\prime}$ ).

Remark 4.2. The set $\mathscr{B}$ computed by Algorithm 1 satisfies the property

$$
\operatorname{SMIN}_{\preccurlyeq}(\mathscr{A}) \subseteq \mathscr{B}=\operatorname{MIN}_{\preccurlyeq}(\mathscr{A}) \subseteq \mathscr{A}
$$

However, in general, the set $\mathscr{B}$ generated by Algorithm 1 may exceed the set $\operatorname{SMIN}_{\preccurlyeq}(\mathscr{A})$.

To overcome the drawback pointed out in Remark 4.2, we present an algorithmic approach for computing $\mathrm{SMIN}_{\preccurlyeq}(\mathscr{A})$ similar to the Jahn-Graef-Younes type methods considered by Eichfelder [2, 3] and also by Köbis and Le [17], involving a final comparison procedure. 


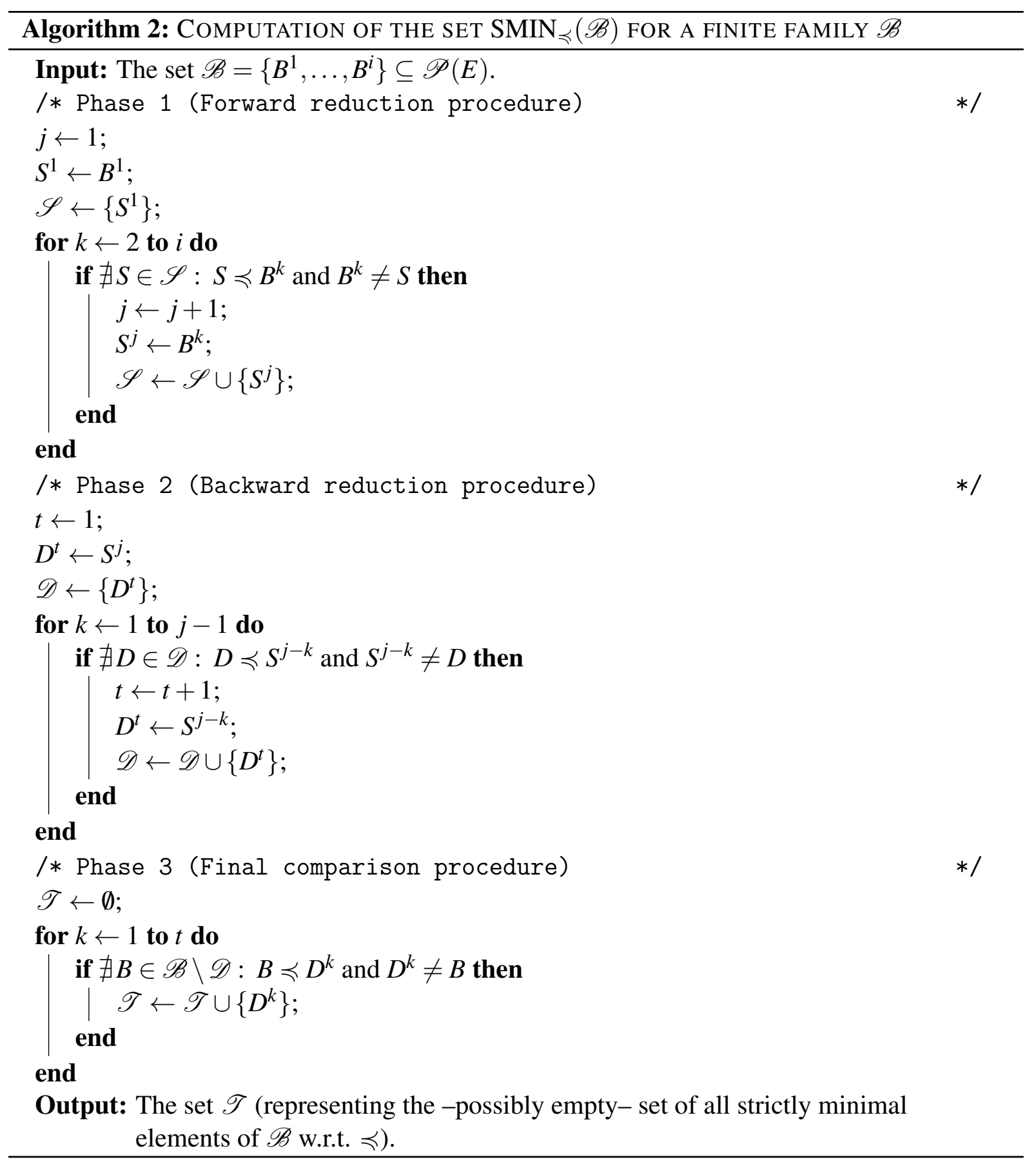

Theorem 4.1. Algorithm 2 generates the set

$$
\mathscr{T}=\operatorname{SMIN}_{\preccurlyeq}(\mathscr{B}) .
$$

Proof. In view of Definition 2.2 (with $\mathscr{B}$ in the role of $\mathscr{A}$ ), it is easy to check that $\operatorname{SMIN}_{\preccurlyeq}(\mathscr{B}) \subseteq$ $\mathscr{T}$. In order to show the reverse inclusion, take some $T \in \mathscr{T}$. Phases 1 and 2 of Algorithm 2 guarantee that $T \in \mathrm{SMIN}_{\preccurlyeq}(\mathscr{D})$, i.e.,

$$
\nexists D \in \mathscr{D}: D \preccurlyeq T \text { and } T \neq D,
$$


while Phase 3 yields

$$
\nexists B \in \mathscr{B} \backslash \mathscr{D}: B \preccurlyeq T \text { and } T \neq B .
$$

Thus, (4.1) and (4.2) show that

$$
\nexists B \in \mathscr{B} \backslash\{T\}: B \preccurlyeq T,
$$

which actually means that $T \in \operatorname{SMIN}_{\preccurlyeq}(\mathscr{B})$ in view of Definition 2.2 (with $\mathscr{B}$ in the role of $\mathscr{A})$.

Remark 4.3. In the worst-case, Algorithm 2 needs to perform at most

$$
\underbrace{(|\mathscr{B}|-1) \cdot|\mathscr{B}| / 2}_{\text {Phase } 1}+\underbrace{(|\mathscr{B}|-1) \cdot|\mathscr{B}| / 2}_{\text {Phase } 2}+\underbrace{|\mathscr{B}| \cdot(|\mathscr{B}|-|\mathscr{T}|)}_{\text {Phase } 3} .
$$

pairwise comparisons of elements w.r.t. $\preccurlyeq$ (where, a comparison of two sets $A, A^{\prime} \in \mathscr{A}$ consists in checking both conditions $A^{\prime} \preccurlyeq A$ and $A \neq A^{\prime}$ ).

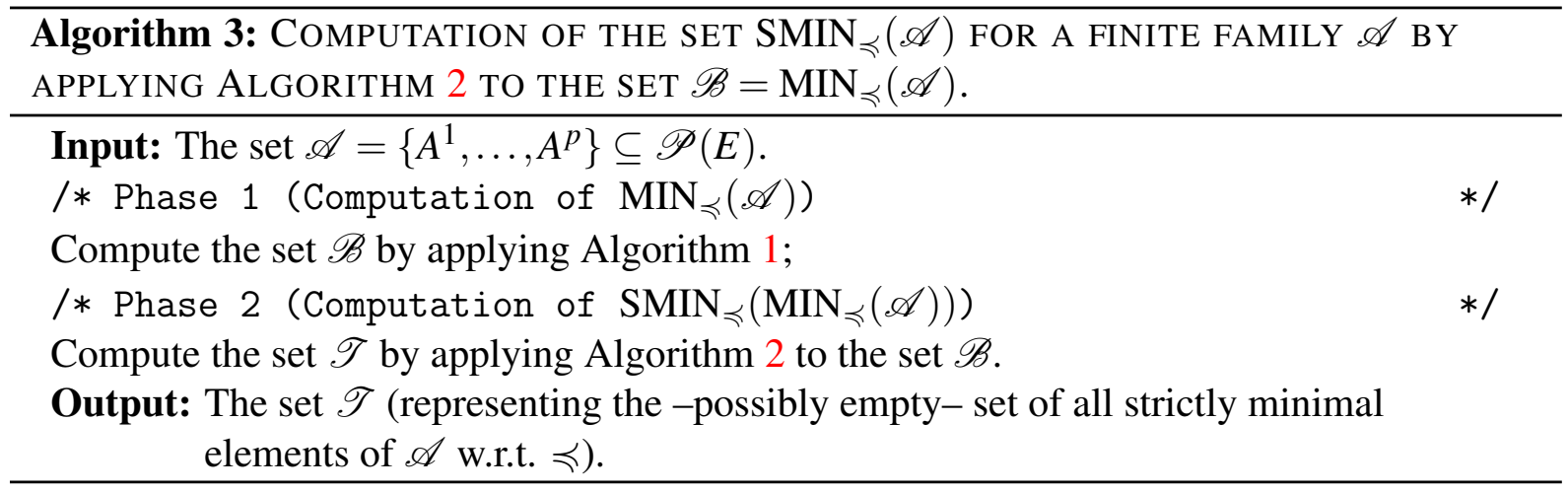

Theorem 4.2. Algorithm 3 generates the set

$$
\mathscr{T}=\mathrm{SMIN}_{\preccurlyeq}(\mathscr{A})=\operatorname{SMIN}_{\preccurlyeq}\left(\mathrm{MIN}_{\preccurlyeq}(\mathscr{A})\right) \text {. }
$$

Proof. As already mentioned in Remark 4.4, the set $\mathrm{MIN}_{\preccurlyeq}(\mathscr{A})$ is nonempty, which ensures the fact that $\mathrm{SMIN}_{\preccurlyeq}(\mathscr{A})=\mathrm{SMIN}_{\preccurlyeq}\left(\mathrm{MIN}_{\preccurlyeq}(\mathscr{A})\right)$ by Theorem 2.1. Thus, the conclusion follows by Theorem 4.1 applied for $\mathscr{B}=\operatorname{MIN}_{\preccurlyeq}(\mathscr{A})$.

Remark 4.4. In view of Remarks 2.2 and 2.3 , the set $\mathscr{B}=\operatorname{MIN}_{\preccurlyeq}(\mathscr{A})$ is externally stable, hence nonempty. Therefore, the sets $\mathscr{S}$ and $\mathscr{D}$ constructed in Phase 2 of Algorithm 3 are nonempty as well. In contrast, the set $\mathscr{T}$ computed in Phase 2 of Algorithm 3 can be empty, as illustrated in the next example.

Example 4.1. Consider $E=\mathbb{R}^{2}, C=\mathbb{R}_{+}^{2}$ and the family $\mathscr{A}=\left\{A^{2}, A^{3}\right\}$, where $A^{2}$ and $A^{3}$ are the rectangles defined in Example 3.1. Since $A^{2} \preccurlyeq_{C}^{\ell} A^{3}, A^{3} \preccurlyeq_{C}^{\ell} A^{2}$ and $A^{2} \neq A^{3}$, it follows that any function $\varphi: \mathscr{A} \rightarrow \mathbb{R}$ is strongly $\preccurlyeq_{C}^{\ell}$-increasing. Therefore, it is easy to see that by applying Algorithm 2 we always have

$$
\mathscr{B}=\mathscr{A}, \mathscr{S}=\left\{A^{k}\right\}, \mathscr{D}=\left\{A^{k}\right\} \text { and } \mathscr{T}=\emptyset
$$

for some $k \in\{2,3\}$. 
Remark 4.5. Whenever $\operatorname{SMIN}_{\preccurlyeq}(\mathscr{A})$ is externally stable (in $\mathscr{A}$ w.r.t. $\preccurlyeq$ ), we have

$$
\operatorname{SMIN}_{\preccurlyeq}(\mathscr{A})=\mathscr{T}=\mathscr{D}=\mathscr{S}=\mathscr{B}=\operatorname{MIN}_{\preccurlyeq}(\mathscr{A})
$$

by Theorem 2.2, where $\mathscr{B}, \mathscr{S}, \mathscr{D}$ and $\mathscr{T}$ represents the sets generated by Algorithm 3 .

Remark 4.6. In the worst-case scenario, our Algorithm 3 needs to perform at most

$$
\underbrace{|\mathscr{B}| \cdot(2|\mathscr{A}|-|\mathscr{B}|-1) / 2}_{\text {Phase } 1}+\underbrace{(|\mathscr{B}|-1) \cdot|\mathscr{B}|+|\mathscr{B}| \cdot(|\mathscr{B}|-|\mathscr{T}|)}_{\text {Phase } 2} .
$$

pairwise comparisons of elements w.r.t. $\preccurlyeq$ (where, a comparison of two sets $A, A^{\prime} \in \mathscr{A}$ consists in checking both conditions $A^{\prime} \preccurlyeq A$ and $A \npreceq A^{\prime}$ for minimality, and both conditions $A^{\prime} \preccurlyeq A$ and $A \neq A^{\prime}$ for strict minimality, respectively). Indeed, in view of Remark 4.1, Phase 1 in Algorithm 3 needs at most $|\mathscr{B}| \cdot(2|\mathscr{A}|-|\mathscr{B}|-1) / 2$ pairwise comparisons of elements w.r.t. $\preccurlyeq$, while, in view of Remark 4.3, Phase 2 needs at most $(|\mathscr{B}|-1) \cdot|\mathscr{B}|+|\mathscr{B}| \cdot(|\mathscr{B}|-|\mathscr{T}|)$ pairwise comparisons in the worst-case.

Taking a closer look on the bounds for the number of pairwise comparisons of elements w.r.t. $\preccurlyeq$ given by (4.3) for Algorithm 2 (applied for $\mathscr{A}$ in the role of $\mathscr{B}$ ), and (4.4) for Algorithm 3, in the case $|\mathscr{B}| \ll|\mathscr{A}|$, it seems that Algorithm 3 is more effective than Algorithm 2 (applied for $\mathscr{A}$ in the role of $\mathscr{B}$ ). This will be confirmed in the next section by numerical experiments.

\section{NUMERICAL EXPERIMENTS}

Throughout this section we restrict our attention to the particular framework of the Euclidean plane $E=\mathbb{R}^{2}$ endowed with the standard ordering cone $C=\mathbb{R}_{+}^{2}$. For certain test families of rectangles, $\mathscr{A}$, we will compute the sets of strictly minimal elements $\operatorname{SMIN}_{\preccurlyeq}(\mathscr{A})$ and $\operatorname{SMIN}_{\preccurlyeq}{ }_{C}^{u}(\mathscr{A})$ by applying the Algorithms 2 and 3 for $\preccurlyeq_{C}^{\ell}$ and $\preccurlyeq_{C}^{u}$ in the role of $\preccurlyeq$.

5.1. Construction of test families of sets and strongly increasing functions. In order to implement and test the Algorithms 2 and 3 we consider finite families of rectangles having one side parallel to the second bisector (possibly degenerated in line segments or singletons). We denote such a family by

$$
\mathscr{A}=\left\{A^{1}, \ldots, A^{p}\right\},
$$

where $p \in \mathbb{N}, p \geq 2$. Actually, for any $i \in\{1, \ldots, p\}$, we can represent $A^{i}$ in the form

$$
A^{i}=a^{i}+\alpha_{i} S^{1}+\beta_{i} S^{2}
$$

for some point $a^{i}=\left(a_{1}^{i}, a_{2}^{i}\right) \in \mathbb{R}^{2}$ and some real numbers $\alpha_{i}, \beta_{i} \geq 0$, where

$$
S^{1}=\{(-x, x) \mid x \in[-1,1]\} \text { and } S^{2}=\{(x, x) \mid x \in[0,1]\} .
$$

Since we will apply Algorithm 3 for $\ell$-type as well as for $u$-type preorder relations, we will construct two functions, $\varphi^{\ell}, \varphi^{u}: \mathscr{A} \rightarrow \mathbb{R}$, that are strongly increasing with respect to $\preccurlyeq_{C}^{\ell}$ and $\preccurlyeq_{C}^{u}$, respectively. To this aim, we follow the approach considered by us in [8] (see also Jahn [12]). For each direction

$$
\lambda \in \Lambda:=\{(1,0),(0,1),(1,1)\}
$$

we define two auxiliary functions $\psi_{\lambda}^{\ell}, \psi_{\lambda}^{u}: \mathscr{A} \rightarrow \mathbb{R}$ for any $A \in \mathscr{A}$ by

$$
\psi_{\lambda}^{\ell}(A)=\min _{a \in A}\langle\lambda, a\rangle \quad \text { and } \quad \psi_{\lambda}^{u}(A)=\max _{a \in A}\langle\lambda, a\rangle .
$$


Lemma 5.1. For any $A, A^{\prime} \in \mathscr{A}$ we have:

$$
\begin{aligned}
& 1^{\circ} A^{\prime} \preccurlyeq_{C}^{\ell} A \Longleftrightarrow \forall \lambda \in \Lambda: \psi_{\lambda}^{\ell}\left(A^{\prime}\right) \leq \psi_{\lambda}^{\ell}(A) . \\
& 2^{\circ} A^{\prime} \preccurlyeq_{C}^{u} A \Longleftrightarrow \forall \lambda \in \Lambda: \psi_{\lambda}^{u}\left(A^{\prime}\right) \leq \psi_{\lambda}^{u}(A) .
\end{aligned}
$$

Proof. Since the sets $A, A^{\prime} \in \mathscr{A}$ are of type (5.1), it is easy to see that for any $\lambda \in \Lambda$ we have

$$
\psi_{\lambda}^{\ell}(A)=\min _{a \in \min _{C}(A)}\langle\lambda, a\rangle \quad \text { and } \quad \psi_{\lambda}^{\ell}\left(A^{\prime}\right)=\min _{a \in \min _{C}\left(A^{\prime}\right)}\langle\lambda, a\rangle .
$$

Moreover, since the sets $\min _{C}(A)$ and $\min _{C}\left(A^{\prime}\right)$ are line segments parallel to the second bisector (possibly degenerated into singletons), it follows by [8, Lem. 5.1] that

$$
\min _{C}\left(A^{\prime}\right) \preccurlyeq_{C}^{\ell} \min _{C}(A) \Longleftrightarrow \forall \lambda \in \Lambda: \psi_{\lambda}^{\ell}\left(A^{\prime}\right) \leq \psi_{\lambda}^{\ell}(A) .
$$

Thus, $1^{\circ}$ follows by the characterization (3.1) in Remark 3.1, since $A$ and $A^{\prime}$ are nonempty compact sets in $E=\mathbb{R}^{2}$, hence they satisfy the domination property w.r.t. $C=\mathbb{R}_{+}^{2}$.

Similarly, for proving $2^{\circ}$, observe that

$$
\psi_{\lambda}^{u}(A)=\max _{a \in \max _{C}(A)}\langle\lambda, a\rangle \quad \text { and } \quad \psi_{\lambda}^{u}\left(A^{\prime}\right)=\max _{a \in \max _{C}\left(A^{\prime}\right)}\langle\lambda, a\rangle .
$$

Then, taking into account that $\min _{C}(A)$ and $\max _{C}(A)$ are also line segments parallel to the second bisector, we deduce by $[8$, Lem. 5.1] that

$$
\max _{C}\left(A^{\prime}\right) \preccurlyeq_{C}^{u} \max _{C}(A) \Longleftrightarrow \forall \lambda \in \Lambda: \psi_{\lambda}^{u}\left(A^{\prime}\right) \leq \psi_{\lambda}^{u}(A)
$$

Thus $2^{\circ}$ follows by (3.2).

Lemma 5.2. For any $A, A^{\prime} \in \mathscr{A}$ we have:

$$
\begin{aligned}
& 1^{\circ} A \aleph_{C}^{\ell} A^{\prime} \Longleftrightarrow \exists \lambda \in \Lambda: \psi_{\lambda}^{\ell}\left(A^{\prime}\right)<\psi_{\lambda}^{\ell}(A) . \\
& 2^{\circ} A \aleph_{C}^{u} A^{\prime} \Longleftrightarrow \exists \lambda \in \Lambda: \psi_{\lambda}^{u}\left(A^{\prime}\right)<\psi_{\lambda}^{u}(A) .
\end{aligned}
$$

Proof. Directly follows by Lemma 5.1.

Consider the functions $\varphi^{\ell}, \varphi^{u}: \mathscr{A} \rightarrow \mathbb{R}$ for any $A \in \mathscr{A}$ by

$$
\varphi^{\ell}(A)=\sum_{\lambda \in \Lambda} \psi_{\lambda}^{\ell}(A) \quad \text { and } \quad \varphi^{u}(A)=\sum_{\lambda \in \Lambda} \psi_{\lambda}^{u}(A)
$$

Following the main lines in the proof of [8, Th. 5.1], it is easy to deduce the following result.

Proposition 5.1. The function $\varphi^{\ell}$ is strongly $\preccurlyeq_{C}^{\ell}$-increasing, while the function $\varphi^{u}$ is strongly $\preccurlyeq_{C}^{u}$-increasing.

Remark 5.1. By (5.1) it follows that, for any $i \in\{1, \ldots, p\}$,

$$
\begin{cases}\psi_{\lambda}^{\ell}\left(A^{i}\right)=a_{1}^{i}-\alpha_{i} \text { and } \psi_{\lambda}^{u}\left(A^{i}\right)=a_{1}^{i}+\alpha_{i}+\beta_{i} \quad \text { if } \quad \lambda=(1,0), \\ \psi_{\lambda}^{\ell}\left(A^{i}\right)=a_{2}^{i}+\alpha_{i} \text { and } \psi_{\lambda}^{u}\left(A^{i}\right)=a_{2}^{i}-\alpha_{i}+\beta_{i} \quad \text { if } \quad \lambda=(0,1) . \\ \psi_{\lambda}^{\ell}\left(A^{i}\right)=a_{1}^{i}+a_{2}^{i} \text { and } \psi_{\lambda}^{u}\left(A^{i}\right)=a_{1}^{i}+a_{2}^{i}+2 \beta_{i} \quad \text { if } \quad \lambda=(1,1) .\end{cases}
$$


5.2. Comparative analysis of our algorithms. In order to compare the efficiency of our Algorithms 2 and 3, we will use large test families of rectangles of type (5.1) following an approach similar to those proposed by Eichfelder and Gerlach [4], and also by us in [8]. More precisely, we construct rectangles of type (5.1) along a planar curve $\gamma:[0, \tau] \rightarrow \mathbb{R}^{2}$ (with $\tau>0$ ). To this aim, we consider a finite number of nodes $0 \leq t_{1}<\cdots<t_{q} \leq \tau(q \in \mathbb{N})$ and three functions $\alpha, \beta, \bar{\beta}:[0, \tau] \rightarrow \mathbb{R}_{+}$. Then, we define the family

$$
\mathscr{A}=\left\{A^{1}, \ldots, A^{p}\right\}
$$

of rectangles of type (5.1), where $p:=2 q$ and

$$
\begin{array}{llll}
a^{i}:=\gamma\left(t_{i}\right), & \alpha_{i}:=\alpha\left(t_{i}\right), & \beta_{i}:=\bar{\beta}\left(t_{i}\right) & \text { for } i \in\{1, \ldots, q\}, \\
a^{i}:=\gamma\left(t_{i-q}\right), & \alpha_{i}:=\alpha\left(t_{i-q}\right), & \beta_{i}:=\underline{\beta}\left(t_{i-q}\right) & \text { for } i \in\{q+1, \ldots, p\},
\end{array}
$$

which means that

$$
A^{i}:= \begin{cases}\gamma\left(t_{i}\right)+\alpha\left(t_{i}\right) S^{1}+\bar{\beta}\left(t_{i}\right) S^{2} & \text { for } i \in\{1, \ldots, q\}, \\ \gamma\left(t_{i-q}\right)+\alpha\left(t_{i-q}\right) S^{1}+\underline{\beta}\left(t_{i-q}\right) S^{2} & \text { for } i \in\{q+1, \ldots, p\} .\end{cases}
$$

In Table 1 we present the numerical results obtained by implementing Algorithms 2 and 3 to four families of sets of type (5.2), with $p \in\{50,10000,50000,100000\}$, where

$$
\gamma(t)=(t,(t / 2) \sin t), \alpha(t)=|\sin t|, \bar{\beta}(t)=|\cos 4 t|, \underline{\beta}(t)=|\sin t||\cos 4 t|,
$$

for any $0 \leq t \leq \tau=4 \pi$, while for every $q \in\{25,5000,25000,50000\}$ the nodes are given by

$$
t_{i}=4 \pi(i-1) /(q-1) \text { for } i \in\{1, \ldots, q\} \text {. }
$$

We mention that in both Algorithms 2 and 3, the pairwise comparisons of sets is performed by means of Lemmas 5.1 and 5.2, where the values of the auxiliary functions $\psi_{\lambda}^{\ell}$ and $\psi_{\lambda}^{u}$ on the elements of $\mathscr{A}$ are a priori computed as indicated in Remark 5.1. Moreover, in Phase 1 of Algorithm 3, we first compute the values of the sorting functions $\varphi^{\ell}$ and $\varphi^{u}$ at the elements of $\mathscr{A}$ by means of the formulae presented in Remark 5.1, and thereafter we sort these values by using the MATLAB function sort. All data presented in Table 1 are obtained in MATLAB 2016a, on a Core i7-8550U CPU, 16GB RAM computer.

As the numerical results listed in Table 1 reveal, both Algorithms 2 and 3 are quite effective even for significantly large $p$, the latter requesting however a much shorter runtime and a lower number of pairwise comparisons of sets w.r.t. $\preccurlyeq_{C}^{\ell}$ and $\preccurlyeq_{C}^{u}$ respectively, due to its first phase.

Finally, we illustrate in Figures 2, 3 and 4 the family $\mathscr{A}$ with $p=50$ along with the corresponding output sets $\operatorname{MIN}_{\preccurlyeq}(\mathscr{A}), \operatorname{SMIN}_{\preccurlyeq_{C}^{\ell}}(\mathscr{A}), \operatorname{MIN}_{\preccurlyeq_{C}^{u}}(\mathscr{A})$ and $\operatorname{SMIN}_{\preccurlyeq_{C}^{u}}(\mathscr{A})$ mentioned in Table 1.

\section{CONCLUSiOns}

Although in Section 5 we have considered a special class of test families and the particular preorder relations $\preccurlyeq_{C}^{\ell}$ and $\preccurlyeq_{C}^{u}$, our algorithms could find interesting applications for other preorder relations known in the literature. It is understood that constructing strongly $\preccurlyeq$-increasing sorting functions for a general preorder relation $\preccurlyeq$ on families of sets with more complex structure than our rectangles (especially, non-polyhedral sets) is a difficult task. However, in Phase 


\begin{tabular}{|c||c|c|c|c|}
\hline$p$ & $\mathbf{5 0}$ & $\mathbf{1 0 0 0 0}$ & $\mathbf{5 0 0 0 0}$ & $\mathbf{1 0 0 0 0 0}$ \\
\hline$\left|\mathrm{MIN}_{\preccurlyeq_{C}^{\ell}}(\mathscr{A})\right|$ & 16 & 2698 & 12156 & 21108 \\
\hline$\left|\mathrm{SMIN}_{\preccurlyeq}(\mathscr{A})\right|$ & 4 & 22 & 90 & 186 \\
\hline$\left|\operatorname{MIN}_{\preccurlyeq_{C}^{u}}(\mathscr{A})\right|$ & 8 & 227 & 1107 & 2207 \\
\hline$\left|\operatorname{SMIN}_{\preccurlyeq_{C}^{u}}(\mathscr{A})\right|$ & 8 & 227 & 1107 & 2203 \\
\hline
\end{tabular}

Algorithm 1

\begin{tabular}{|c||c|c|c|c|}
\hline Runtime for MIN $\preccurlyeq_{C}^{\ell}(\mathscr{A})$ & 0.0107 & 8.7254 & 194.9568 & 616.5782 \\
\hline \# pairwise comparison w.r.t. $\preccurlyeq_{C}^{\ell}$ & 242 & 6828053 & 154550044 & 549491318 \\
\hline Runtime for MIN $\preccurlyeq_{C}^{u}(\mathscr{A})$ & 0.0067 & 0.3696 & 5.9141 & 19.5573 \\
\hline \# pairwise comparison w.r.t. $\preccurlyeq_{C}^{u}$ & 121 & 201182 & 4547514 & 17843076 \\
\hline
\end{tabular}

Algorithm 2 (applied for $\mathscr{A}$ in the role of $\mathscr{B}$ )

\begin{tabular}{|c|c|c|c|c|}
\hline Runtime for SMIN $\preccurlyeq_{C}(\mathscr{A})$ & 0.0205 & 15.7443 & 373.4251 & 1263.9630 \\
\hline \# pairwise comparison w.r.t. $\preccurlyeq_{C}^{\ell}$ & 507 & 12867361 & 302182730 & 1102711765 \\
\hline Runtime for SMIN $_{\preccurlyeq u}(\mathscr{A})$ & 0.0124 & 6.0336 & 126.0268 & 469.9523 \\
\hline \# pairwise comparison w.r.t. $\preccurlyeq_{C}^{u}$ & 639 & 4173417 & 102261836 & 4082355860 \\
\hline
\end{tabular}

Algorithm 3

\begin{tabular}{|c|c|c|c|c|}
\hline Runtime for SMIN $_{\preccurlyeq_{C}(\mathscr{A})}$ & 0.0198 & 13.2452 & 287.6983 & 902.4062 \\
\hline \# pairwise comparison w.r.t. $\preccurlyeq_{C}^{\ell}$ & 406 & 10509315 & 229218876 & 775095762 \\
\hline Runtime for SMIN $_{\preccurlyeq c}(\mathscr{A})$ & 0.0142 & 0.4332 & 6.9132 & 26.7075 \\
\hline \# pairwise comparison w.r.t. $\preccurlyeq_{C}^{u}$ & 177 & 252484 & 5771856 & 22708060 \\
\hline
\end{tabular}

TABlE 1. Computational study for the four test families $\mathscr{A}$ in Section 5.2.

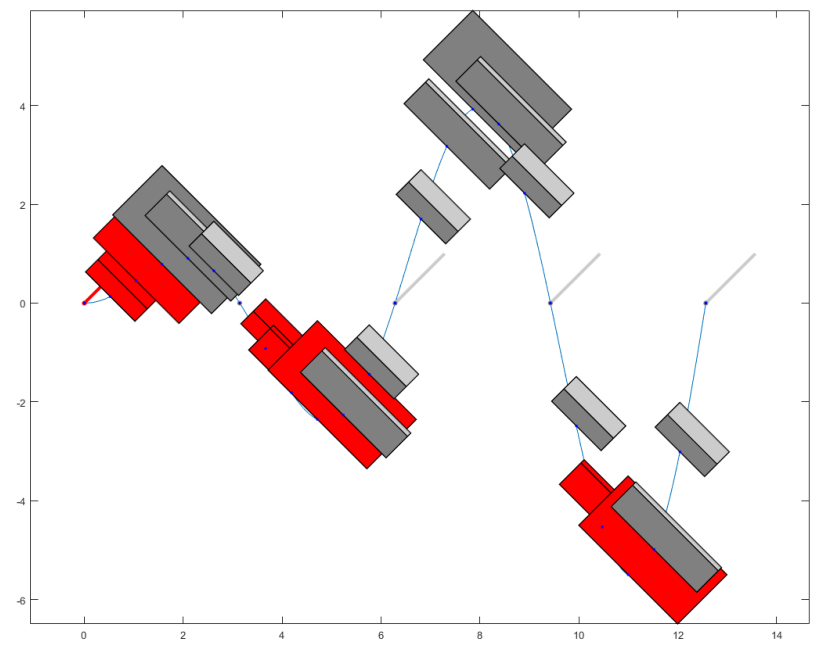

FIgURE 2. The set $\operatorname{MIN}_{\preccurlyeq_{C}^{\ell}}(\mathscr{A})$ mentioned in Table 1 for $p=50$ (red color).

1 of Algorithm 3 one can use alternative methods for computing the set $\mathrm{MIN}_{\preccurlyeq}(\mathscr{A})$ instead of Algorithm 1, as for instance our "Generalized Jahn-Graef-Younes Method" [8], which does not 


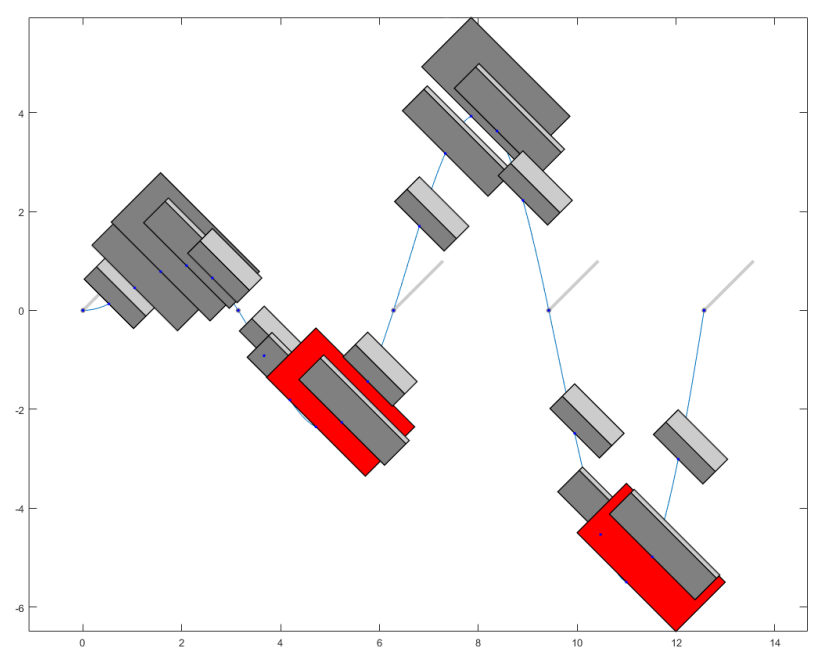

FIGURE 3. The set $\mathrm{SMIN}_{\preccurlyeq_{C}^{\ell}}(\mathscr{A})$ mentioned in Table 1 for $p=50$ (red color).

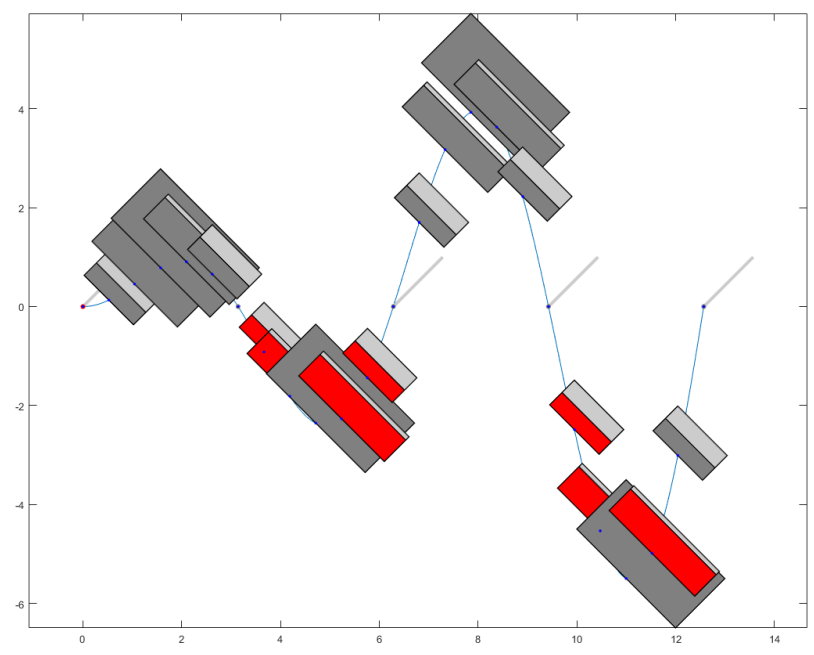

FIgURE 4. The set $\mathrm{SMIN}_{\preccurlyeq} \preccurlyeq_{C}^{u}(\mathscr{A})=\operatorname{MIN}_{\preccurlyeq}{ }_{C}^{u}(\mathscr{A})$ mentioned in Table 1 for $p=50$ (red color).

involve sorting functions. Another interesting topic for further research would be to use our algorithms as intermediate procedures within certain population-based algorithms for solving continuous set optimization problems.

\section{Acknowledgments}

Nicolae Popovici's research was partially supported by a grant of the Romanian Ministry of Research and Innovation, CNCS-UEFISCDI, project number PN-III-P4-ID-PCE-2016-0190, within PNCDI III.

\section{REFERENCES}

[1] G. Eichfelder, Vector optimization in medical engineering, In: P.M. Pardalos, T.M. Rassias (Eds.), Mathematics without boundaries. Surveys in interdisciplinary research, pp. 181-215, Springer, New York, 2014. 
[2] G. Eichfelder, Numerical procedures in multiobjective optimization with variable ordering structures, J. Optim. Theory Appl. 162 (2014), 489-514.

[3] G. Eichfelder, Variable Ordering Structures in Vector Optimization, Springer, Berlin, Heidelberg, 2014.

[4] G. Eichfelder, T. Gerlach, On classes of set optimization problems which are reducible to vector optimization problems and its impact on numerical test instances, Chapter 10 in: A. Khan, E. Köbis, Chr. Tammer (Eds.), Variational Analysis and Set Optimization, pp. 265-290, CRC Press, 2019.

[5] G. Eichfelder, M. Pilecka, Set approach for set optimization with variable ordering structures, Part I: Set relations and relationship to vector approach, J. Optim. Theory Appl. 171 (2016), 931-946.

[6] G. Eichfelder, M. Pilecka, Set approach for set optimization with variable ordering structures, Part II: Scalarization approaches, J. Optim. Theory Appl. 171 (2016), 947-963.

[7] A. Göpfert, H. Riahi, C. Tammer, C. Zălinescu, Variational Methods in Partially Ordered Spaces, Springer, New York, 2003.

[8] C. Günther, E. Köbis, N. Popovici, Computing minimal elements of finite families of sets w.r.t. preorder relations in set optimization, J. Appl. Numer. Optim. 1 (2019), 131-144.

[9] C. Günther, N. Popovici, New algorithms for discrete vector optimization based on the Graef-Younes method and cone-monotone sorting functions, Optimization 67 (2018), 975-1003.

[10] J. Jahn, Multiobjective search algorithm with subdivision technique, Comput. Optim. Appl. 35 (2006), 161175.

[11] J. Jahn, Vector Optimization - Introduction, Theory, and Extensions, Springer, Berlin, Heidelberg, 2011.

[12] J. Jahn, Vectorization in set optimization, J. Optim. Theory Appl. 167 (2015), 783-795.

[13] J. Jahn, T.X.D. Ha, New order relations in set optimization, J. Optim. Theory Appl. 148 (2011), 209-236.

[14] J. Jahn, U. Rathje, Graef-Younes method with backward iteration, In: Chr. Tammer, K. Winkler, K.H. Küfer, H. Rommelfanger (Eds.), Multicriteria decision making and fuzzy systems - Theory, methods and applications, pp. 75-81, Shaker Verlag, Aachen, 2006.

[15] A. Khan, C. Tammer, C. Zălinescu, Set-Valued Optimization - An Introduction with Applications, Springer, Berlin, Heidelberg, 2015.

[16] E. Köbis, D. Kuroiwa, C. Tammer, Generalized set order relations and their numerical treatment, Appl. Anal. Optim. 1 (2017), 45-65.

[17] E. Köbis, Th.T. Le, Numerical procedures for obtaining strong, strict and ideal minimal solutions of set optimization problems, Appl. Anal. Optim. 2 (2018), 423-440.

[18] D. Kuroiwa, The natural criteria in set-valued optimization. RIMS Kokyuroku 1031 (1998), 85-90.

[19] D.T. Luc, Theory of Vector Optimization, Springer, Berlin, 1989.

[20] V.V. Podinovskiur, V.D. Nogin, Pareto optimal solutions of multicriteria optimization problems (in Russian), Nauka, Moscow, 1982.

[21] K. Seto, D. Kuroiwa, N. Popovici, A systematization of convexity and quasiconvexity concepts for set-valued maps, defined by $l$-type and $u$-type preorder relations, Optimization 67 (2018), 1077-1094.

[22] Y.M. Younes, Studies on discrete vector optimization, Dissertation, University of Demiatta, Egypt, 1993. 